University of Nebraska - Lincoln

DigitalCommons@University of Nebraska - Lincoln

Faculty Publications - Textiles, Merchandising and Fashion Design

Textiles, Merchandising and Fashion Design,

Department of

2013

Structure and Properties of Cocoons and Silk Fibers Produced by Attacus atlas

Narendra Reddy

University of Nebraska - Lincoln, nreddy3@unl.edu

Yi Zhao

University of Nebraska-Lincoln, yzhao8@unl.edu

Yiqi Yang

University of Nebraska-Lincoln, yyang2@unl.edu

Follow this and additional works at: https://digitalcommons.unl.edu/textiles_facpub

Part of the Biology and Biomimetic Materials Commons, Bioresource and Agricultural Engineering Commons, Mechanics of Materials Commons, and the Polymer and Organic Materials Commons

Reddy, Narendra; Zhao, Yi; and Yang, Yiqi, "Structure and Properties of Cocoons and Silk Fibers Produced by Attacus atlas" (2013). Faculty Publications - Textiles, Merchandising and Fashion Design. 32. https://digitalcommons.unl.edu/textiles_facpub/32

This Article is brought to you for free and open access by the Textiles, Merchandising and Fashion Design, Department of at DigitalCommons@University of Nebraska - Lincoln. It has been accepted for inclusion in Faculty Publications - Textiles, Merchandising and Fashion Design by an authorized administrator of DigitalCommons@University of Nebraska - Lincoln. 
Published in Journal of Polymers and the Environment 21 (2013) 21:16-23; doi: 10.1007/s10924-012-0549-8

Copyright (C) Springer Science+Business Media New York. Used by permission.

Published online November 8, 2012

\title{
Structure and Properties of Cocoons and Silk Fibers Produced by Attacus atlas
}

\author{
Narendra Reddy, ${ }^{1}$ Yi Zhao, ${ }^{1}$ and Yiqi Yang ${ }^{1,2,3}$ \\ 1. Department of Textiles, Merchandising \& Fashion Design, University of Nebraska-Lincoln, \\ 234 HECO Building, East Campus, Lincoln, NE 68583-0802, USA \\ 2. Department of Biological Systems Engineering, University of Nebraska-Lincoln, \\ 234 HECO Building, East Campus, Lincoln, NE 68583-0802, USA \\ 3. Nebraska Center for Materials and Nanoscience, University of Nebraska-Lincoln, \\ 234 HECO Building, East Campus, Lincoln, NE 68583-0802, USA \\ Corresponding author - Yiqi Yang, email yyang2@unl.edu
}

\begin{abstract}
Silk fibers in the three layers of Attacus atlas (A. atlas) cocoons have morphological structure and tensile properties similar to that of Bombyx mori silk. Attempts are being made to produce silk for commercial applications from cocoons of relatively unknown wild insects due to the unique properties of the fibers and as a source of income and employment. In this research, $A$. atlas cocoons were used to study the chemical composition, morphology, physical structure and tensile properties of the silk fibers in the cocoons and ability of the fibers to support the attachment and proliferation of mouse fibroblast cells. It was found that $A$. atlas cocoons consists of outer, intermediate and inner layer with average breaking tenacity of $4.1,4.3$, and $3.6 \mathrm{~g} / \mathrm{den}$, respectively similar to that of $B$. mori silk (4.3-5.2 g/den). The heavier cocoons, less restrictive rearing conditions and good properties of the fibers compared to B. mori silk makes $A$. atlas a potential alternative to common silks for commercial scale silk production. A. atlas fibers had about $80 \%$ higher optical densities of cells and extensive growth of F-actin compared to B. mori silk fibers.
\end{abstract}

Keywords: Wild silks, Attacus atlas, Biocompatibility, Tensile properties, Bombyx mori

\section{Introduction}

Silk fibers and protein based materials, in general, are preferred for biomedical, textile and biotechnology industries [1-5]. In addition to the common Bombyx mori silks, silks from several other sources such as spider silks and non-domesticated silk worms such as Antheraea pernyi are being studied for potential use in various applications [6, 7]. For instance, the exceptional tensile strength of spider silk has been well documented and several attempts have to made to produce regenerated spider silk [8]. Similarly, it has been found that fibroin from wild silks has better potential for medical applications compared to fibroin from B. mori silk [9]. Recent studies also suggest that, contrary to popular belief, silk sericin also has the potential for medical applications [9, 10]. Although a wide variety of insects have been known to produce silk containing cocoons, only a few non-mulberry silks have been studied for their structure, properties and potential applications. Insects belonging to the saturniidae family are one of the largest group of insects known to produce cocoons containing silk [11]. Among the numerous saturniidae insects, Antheraea mylitta (Indian tasar), Antheraea pernyi (Chinese tasar), and Philosamia ricini (Eri) have been extensively studied for their structure, properties and applications $[12,13]$. Silk from these wild insects is being processed for commercial applications. However, the common non-mulberry wild silk fibers are much coarser 
than the B. mori silk [14]. Therefore, the wild silk fibers have limited applications compared to $B$. mori silk fibers. In addition to the three saturniidae insects $A$. mylitta, $A$. pernyi, and $P$. ricini, other insects also belonging to the saturniidae family such as Eupackardia callata, Rothschildia lebeau, Hyalophora gloveri, Actias luna, Coscinocera hercules, Antheraea oculea, and Copaxa multifenestrata also produce cocoons containing silk. Fibers from $C$. hercules were found to have properties similar to that of B. mori silk whereas fibers from $C$. multifenestrata were much coarser and had low breaking tenacity and breaking elongation compared to B. mori silk [15]. Likewise, Hylaphora cecropia cocoons were reported to consist of three layers with properties similar to that of B. mori silk [16]. However, silk produced by bag worms was found to be weak and with considerably different amino acids than those found in common silks [17].

In addition to obtaining fibers with unique properties, utilizing cocoons from wild insects for commercial silk production will provide economic benefits. Since the wild insects are located in forest and tribal areas, developing silks from the wild insects will provide income to the indigenous people. Therefore, efforts are being made to rear wild silks for commercial silk production [18, 19].

Attacus atlas belongs to the saturniidae family which includes the common wild silks currently used for commercial silk production. Attacus atlas insects are primarily found in the tropical forests and are considered to the largest Lepidoptera insects based on their wing span which exceeds 65 square inches [20]. These insects produce silk containing cocoons and the color of the cocoons varies from silver to black depending on the host plant. Attacus atlas cocoons have been processed to develop yarns and fabrics. In another report, tensile properties of the A. atlas silk fibers submerged in liquid media have also been studied [21]. It was reported that water disrupted the protein-protein hydrogen bonds whereas ethanol enhanced the protein-protein hydrogen bonds. Significant differences in tensile properties of $A$. atlas and spider silk were observed even though they had similar amino acid composition. The previous research by Perez-Rigueiro was focused on understanding the tensile properties of the A. atlas silk fibers in liquid media [21]. In this research, we have studied the properties of the A. atlas cocoons and the silk fibers from the three layers in A. atlas cocoons have been characterized for their structure and properties in comparison to B. mori and common wild silks.

\section{Experimental}

Materials

Attacus atlas cocoons were supplied by Reiman Gardens in Ames, Iowa. Chemicals used for the study were purchased from VWR international (Bristol, CT).

\section{Degumming}

The $A$. atlas cocoons were separated into three layers by hand. The three layers were separately degummed using ethylenediamene and sodium carbonate. Cocoon layers were treated in $10 \%$ ethylenediamine and $0.5 \%$ sodium carbonate for $1 \mathrm{~h}$ at $80{ }^{\circ} \mathrm{C}$ with a solution to cocoon ratio of 20:1. Later, cocoons were washed in water and retreated in the ethylenediamine and sodium carbonate solution at $80{ }^{\circ} \mathrm{C}$ for $15 \mathrm{~min}$ to complete the degumming and obtain loose fibers. Degummed silk was thoroughly washed in water and dried under ambient conditions.

Morphology

Images of the three different layers of $A$. atlas cocoons were collected using a digital camera. Surface features of the different layers in the cocoons and the longitudinal and cross-sectional features of the degummed fibers were observed using a variable pressure scanning electron microscope (VP-SEM). Samples were sputter coated with gold palladium and observed in the SEM at a voltage of $20 \mathrm{kV}$.

\section{Composition}

The type and percentage of amino acids in the A. atlas fibers was determined using a Hitachi L-8800A amino acid analyzer. Norleucine was used as the internal standard for both the samples and the control for amino acid analysis. Samples were first evaporated to dryness in a speedvac and later hydrolyzed in liquid $6 \mathrm{~N} \mathrm{HCl}$ under argon atmosphere. After $20 \mathrm{~h}$ of hydrolysis at $110^{\circ} \mathrm{C}$, the samples were evaporated to dryness, then redissolved in $200 \mu \mathrm{L}$ of $0.02 \mathrm{~N} \mathrm{HCl}$. Fifty microliters was injected automatically onto the Hitachi Amino Acid Analyzer to determine the amino acid type and content. In data analysis, correction was made to the amount of the internal standard, to minimize dilutional errors.

\section{Physical Structure}

Physical structure of the A. atlas fibers was determined in terms of \% crystallinity and peak positions using a

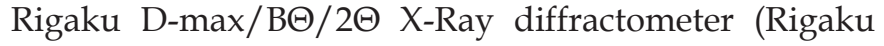
Americas, Woodlands, TX) with Bragg-Brentano parafocusing geometry, a diffracted beam monochromator, and a copper target X-ray tube set to $40 \mathrm{kV}$ and $30 \mathrm{~mA}$. Fibers were powdered and compressed to form pellets for the X-ray measurements. Powdered samples were used for the X-ray analysis to eliminate any preferred orientation of the crystals to the fiber axes. This is a common practice in obtaining $X$-ray diffraction data for fibers. Diffraction intensities were collected for $2 \theta$ val- 
ues ranging from 5 to $40^{\circ}$. The $\%$ crystallinity of the fiber was obtained by integrating the area under the crystalline peaks after subtracting the background and air scatter using the program MICROCAL ORIGIN.

\section{Tensile Properties and Moisture Regain}

Samples were conditioned for at least $24 \mathrm{~h}$ under the standard testing conditions of $21^{\circ} \mathrm{C}$ and $65 \%$ relative humidity before testing. Fineness of the fibers was calculated in terms of denier (weight in grams per $9,000 \mathrm{~m}$ of the fibers) by weighing a known length of the fibers. The tensile properties of the fibers from the outer, intermediate and inner layers were determined separately on an Instron tensile tester using a gauge length of 1 inch and cross head speed of $18 \mathrm{~mm} /$ minute. At least 50 fibers from ten different cocoons were tested and the average and standard deviations were obtained. Moisture regain of the fibers was determined according to ASTM standard method 2654 using standard conditions of $21^{\circ} \mathrm{C}$ and $65 \%$ relative humidity.

\section{Cell Culture and Biocompatibility}

Ability of $A$. atlas fibers to support the attachment and growth of mouse fibroblast cells was studied in comparison to the common B. mori silk. Silk fibers were compressed into a sheet and sterilized in an autoclave at $120{ }^{\circ} \mathrm{C}$. Samples were accurately weighted and placed in a 24-well culture plate and incubated with NIH3T3 mouse fibroblast cells at a concentration of $1.5 \times 105$ cells $\mathrm{mL}^{-1}$ DMEM media containing $4 \mathrm{mM}$ l-glutamine, $10 \%$ calf serum, and $1.0 \%$ of $104 \mu \mathrm{g} / \mathrm{mL}$ penicillin/ streptomycin were added into each well and the plates were incubated at $37^{\circ} \mathrm{C}$ and $5 \% \mathrm{CO} 2$ up to 11 days. Media was refreshed every 3 days. After the desired length of incubation, the samples were transferred to a new culture plate and the unattached cells were removed by rinsing with phosphate buffered saline. Metabolic activities of the cells on the silk fibers were measured using a MTS assay. To perform the assay, about $0.5 \mathrm{~mL}$ per well of $20 \%$ MTS solution in DMEM media was added into each well and incubated for $3 \mathrm{~h}$. After $3 \mathrm{~h}, 150 \mu \mathrm{L}$ of DMEM was collected into a 96-well plate and the absorbance of the solution was read on a Mutiwell plate reader (Thermoscientific Model: Multiskan) at a wavelength of $490 \mathrm{~nm}$ to obtain the optical densities. Blank samples without any cells were also tested and used as control. At least four samples were tested for each time point and the experiments were repeated twice. The average optical density of the cells with \pm one standard deviation are reported.
Fibers after cell culture were also observed in a Confocal laser scanning microscope (Olympus, Model: FV500) to detect the extent of cell growth and spreading of the cytoskeleton. For the confocal study, the cells were fixed using $4 \%$ paraformaldehyde and then stained with the fluorescent dyes (Phallodin 633 and Hoechst 33342) to stain the cell and nuclei in red and blue, respectively.

\section{Results and Discussion}

\section{Construction of the Cocoon}

Attacus atlas cocoons used in this research had an average weight of $1.21 \mathrm{~g}$, much higher than that of $B$. mori (640 milligrams) and $P$. ricin $(840 \mathrm{mg}$ ) cocoons but lower than that of the A. mylitta $(3.4 \mathrm{~g})$ cocoons $[12,13]$. The $A$. atlas cocoons are constructed in three distinct layers. The outer layer was loosely attached and was paper-like as seen from Figure 1a and had a thickness of $0.5 \pm 0.2 \mathrm{~mm}$ and accounted for approximately $23 \%$ of the total weight of the cocoon. In comparison, middle or intermediate layer (Figure $1 \mathrm{~b}$ ) with a thickness of $0.6 \pm 0.2 \mathrm{~mm}$ formed the bulk of the cocoons and accounted for $43 \%$ of the weight of the cocoon. The intermediate layer contains most of the fibers and was loosely connected to the outer layer but very tightly connected to the inner layer. Innermost layer seen in Figure 1c accounted for approximately $34 \%$ of the weight of the cocoon and had an average thickness of $0.20 \pm 0.09 \mathrm{~mm}$. As seen from Figure 1c, the size of the layers decreased progressively from the outer layer to the inner layer. It is reported that the caterpillar decreases its size as it builds the cocoons from outside to inside making the cocoons compact with the inner most layer being the smallest.

\section{Effect of Degumming}

Degumming resulted in removal of the gums (sericin) and disintegration of the cocoons into lustrous fibers. In addition to sericin, wild silks cocoons are mineralized and contain considerable amounts of calcium oxalate crystals that affect degumming and the properties of the cocoons. It has been reported that treating with ethylenediamine is effective in removing calcium oxalate from the cocoon. Among the three layers of the cocoons, the outer layer of $A$. atlas cocoons had the highest degumming loss of $22 \%$ followed by the inner and intermediate layers with degumming loss of 10 and $7.3 \%$, respectively. Relatively stronger conditions are required to degum wild silk fibers compared to $B$. mori silk fibers due to the chemical interactions between wild silk sericins and inorganic components and the minerals present in the cocoons [22]. B. mori silk fibers are reported to 

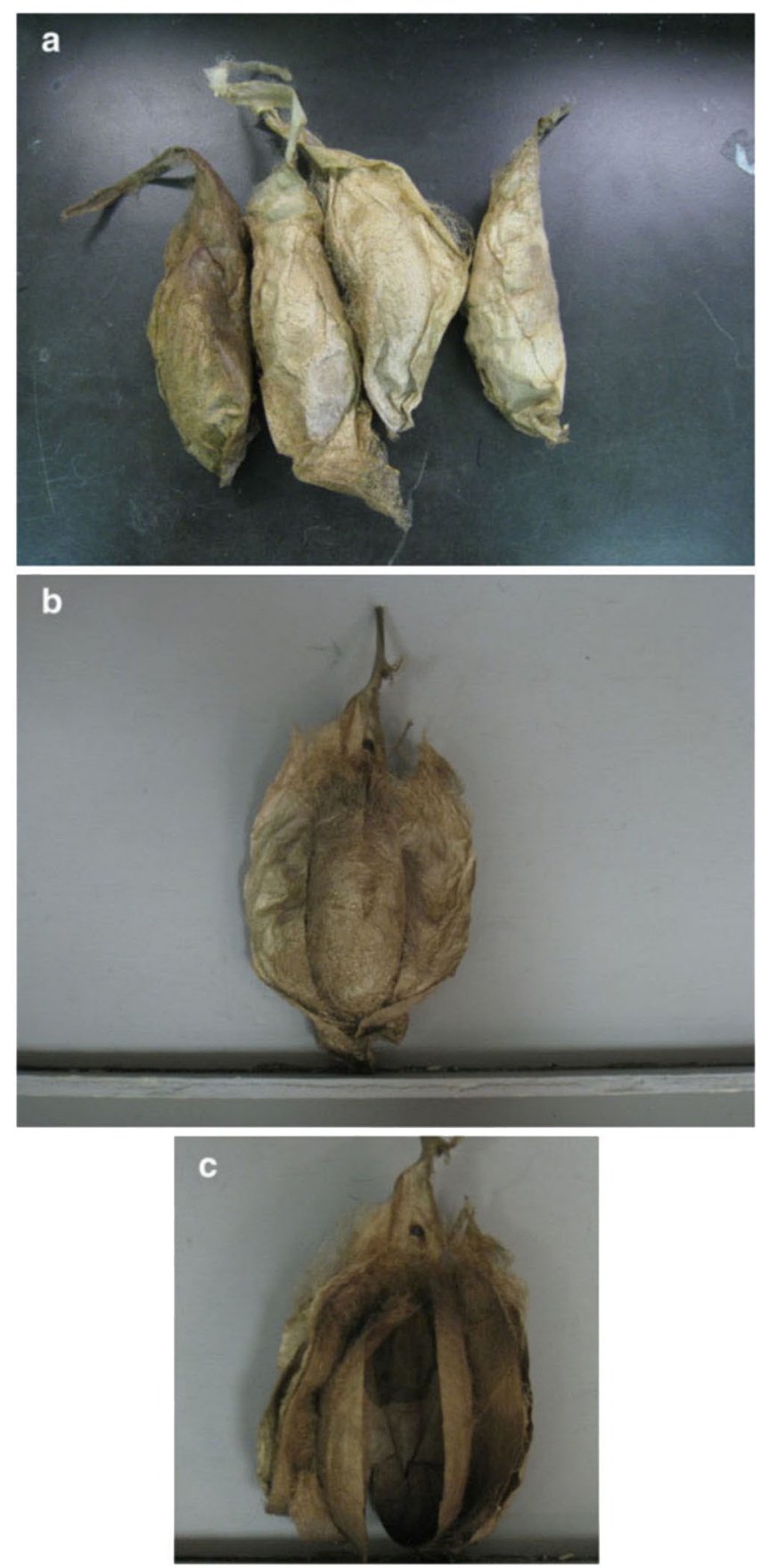

Figure 1. Structure of the $A$. atlas cocoons. a shows an intact cocoon, $\mathrm{b}$ reveals the fiber (intermediate) layer after the outer most layer has been cut open and $\mathrm{c}$ shows the three layers (outer, intermediate and inner layer) in the cocoons.

have sericin content ranging from 20 to $30 \%$ and mild alkali at $90{ }^{\circ} \mathrm{C}$ and degumming without ethylenediamine or other chelating agents are normally adequate for B. mori silk [22]. It has also been reported that the sericin content in cocoons progressively decreases from the outside layers to the inside layers similar to that observed for the A. atlas cocoons in this research [23]. The sericin content in the $B$. mori cocoons varied from about $30 \%$ in the outer layer to about $20 \%$ in the inner most layer [23]. Although sericin needs to be removed from silk fibers to make the fibers lustrous, sericin proteins are found to have unique properties and used for applications in various areas. Sericin resists oxidation, has antibacterial and anti-UV resistant properties [22, 24]. Silk sericin has been used for medical, pharmaceutical and cosmetic industries [24, 25].

\section{Morphology of the Layers in the A. atlas Cocoons}

SEM images of the three layers of $A$. atlas cocoons are shown in Figure 2. As seen from the figure, the outer layer (Figure 2a) has a relatively open construction compared to the intermediate (Figure $2 b$ ) and inner layers (Figure 2c). The inner layer is very compact and has fibers much closer to each other than the fibers in the outer and intermediate layers as seen from Figure 2c. As seen from Figs. 2a to c, the A. atlas caterpillar extrudes two adjacent fibers simultaneously. The two adjacent fibers are attached to each other by glues in the intermediate and inner layers but the outer layer has fibers that are not as extensively attached to each other as fibers in the other two layers. Inner layer also had fibers with slightly lower diameters than the fibers in the outer and intermediate layers. Average diameter of a single fiber in the inner layer was $17 \pm 5 \mu \mathrm{m}$ and those in the outer and intermediate layer was $19 \pm 4$ and $20 \pm 4 \mu \mathrm{m}$, respectively. It has been reported that the filaments in $A$. atlas consist of microfibrils of about $1 \mu \mathrm{m}$ in diameter and embedded in a soft matrix [26].

\section{Composition}

Silk produced by $A$. atlas has considerably different composition of amino acids than the common silks as seen from Table 1. Among the major amino acids, A. atlas has more than twice the tyrosine, about $53 \%$ higher alanine but about $50 \%$ lower glycine and $56 \%$ lower serine content than B. mori silk. However, the alanine and glycine content in A. atlas silk is similar to that of the wild silks. The glycine/alanine ratio for $A$. atlas silk is 0.5 , much lower than that of mulberry (1.5) and the common wild silks (0.8). Amount of glycine and alanine determines the crystallographic form of the proteins [12, 13]. The lower ratio glycine/alanine in A. atlas silk suggests that $A$. atlas silk could have a considerably different crystallographic structure compared to mulberry and wild silks. A. atlas silk has much higher amounts of hydrophobic amino acids (67 \%) compared to the hydrophilic amino acids $(23 \%)$ indicating that the A. atlas silk may have lower moisture absorption and lower sorption of dyes and chemicals if the crystal structures and $\%$ crystallinity are similar. 

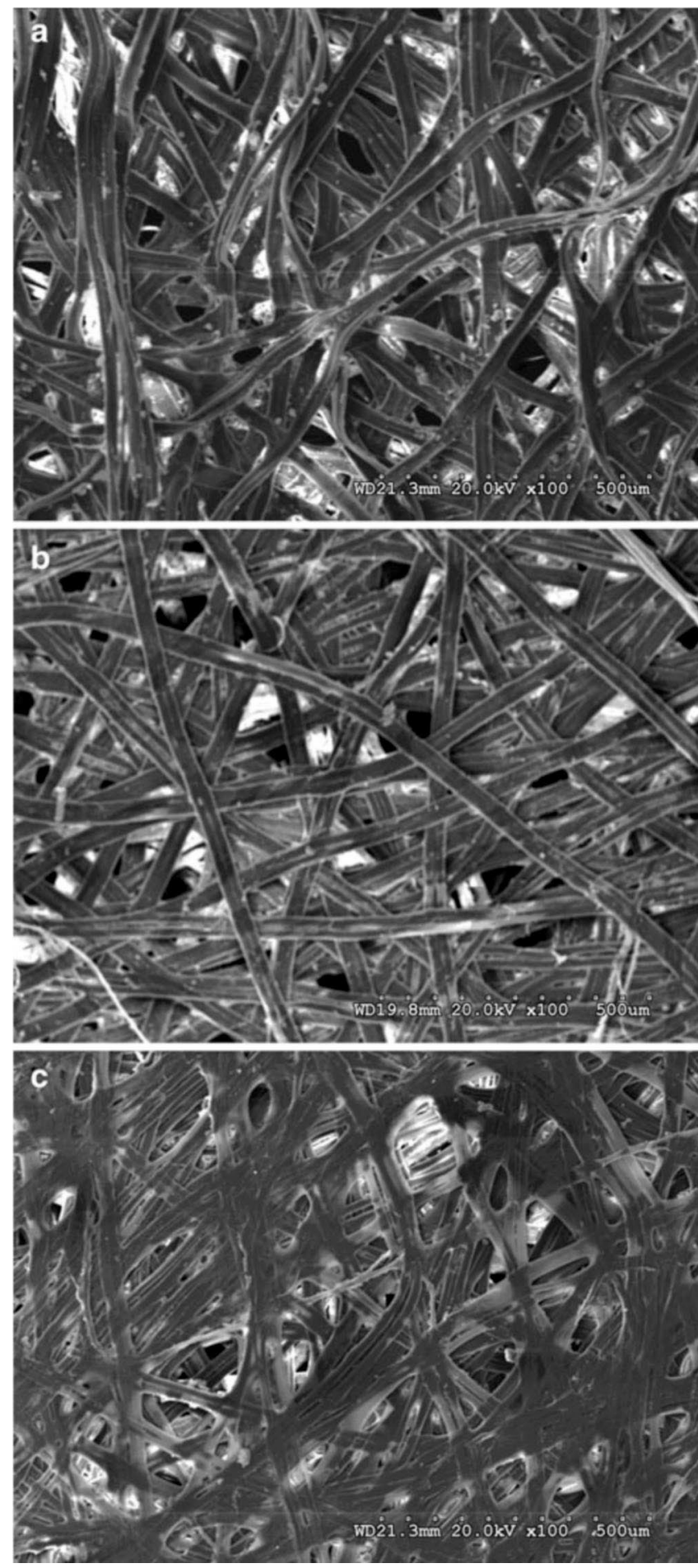

Figure 2. SEM images of the outer (a), intermediate (b) and inner (c) layers in the $A$. atlas cocoons before degumming. The white particles in the cocoons are the calcium oxalate crystals.

A previous report studying the properties of A. atlas silk fibers immersed in liquid media has reported considerably different amino acid composition [21]. In that report, A. atlas silk was reported to contain $40.7 \%$ glycine, $18 \%$ alanine and $8.8 \%$ serine as the major amino
Table 1. Comparison of the amino acid composition of the silk from the fiber layer in A. atlas cocoons with B. mori and three varieties of common wild silks

\begin{tabular}{llllll}
\hline Amino acids & \multicolumn{5}{c}{$\%$ Amino acids } \\
\hline Alanine & 45.0 & 29.4 & 34.1 & 34.7 & 36.3 \\
Tyrosine & 11.4 & 5.2 & 6.8 & 5.1 & 5.8 \\
Glycine & 22.2 & 44.6 & 27.7 & 28.4 & 29.4 \\
Serine & 6.8 & 12.1 & 9.9 & 9.1 & 8.9 \\
Aspartic acid & 3.2 & 1.3 & 6.1 & 5.0 & 3.9 \\
Arginine & 2.2 & 0.5 & 5.0 & 4.7 & 4.1 \\
Glutamic acid & 3.6 & 1.8 & 1.3 & 1.4 & 1.3 \\
Histidine & 2.2 & 0.1 & 0.8 & 0.7 & 0.8 \\
\hline
\end{tabular}

Data for B. mori and the wild silks are from [11-13]

acids compared to $22.2,45$ and $6.8 \%$, respectively in this research. However, the total glycine, alanine and serine in $A$. atlas previously reported and found in this research are similar at 74 and $67.5 \%$, respectively. In the previous study by Perez-Rigueiro, it was suggested that glycine, alanine, serine and threonine exist in the crystalline region and all other amino acids exist in the amorphous region. The ratio of amino acids in the crystalline and non-crystalline regions was called the disorder ratio and related to the properties of the fibers [21]. The variations in the amino acid contents of $A$. atlas silk in the previous report and current research should be due to habitat of the insects, rearing conditions and differences in the methods used to determine the amino acids.

\section{Physical Structure}

X-ray diffractogram of $A$. atlas fibers has been compared to B. mori silk fibers in Figure 3. A. atlas fibers had two strong distinct diffracting peaks at 17 and $20^{\circ}$ corresponding to the reflections from the 002 and 201 planes whereas $B$. mori silk had a single diffracting peak at about $20.6^{\circ}$ corresponding to the 201 plane reflection $[12,13]$. However, other non-mulberry silks also showed the presence of two diffracting peaks at 17 and $20^{\circ}$. It has been suggested that the nature of crystals in silk is dependent on the alanine/glycine ratio. The higher alanine content provides a better crystallographic form for the $A$. atlas silk compared to $B$. mori silk. The \% crystallinity of A. atlas fibers was $32.8 \%$ close to the high end reported for B. mori (20-41\%) silk fibers [12, 13].

\section{Morphology of the Fibers}

Morphology of the A. atlas fibers after degumming are shown in Figs. 4 and 5. Longitudinally, the fibers have a clean and smooth surface as seen from Figure 4. Figure $5 \mathrm{a}$ and $\mathrm{b}$ reveals that the fibers are flat and rib- 


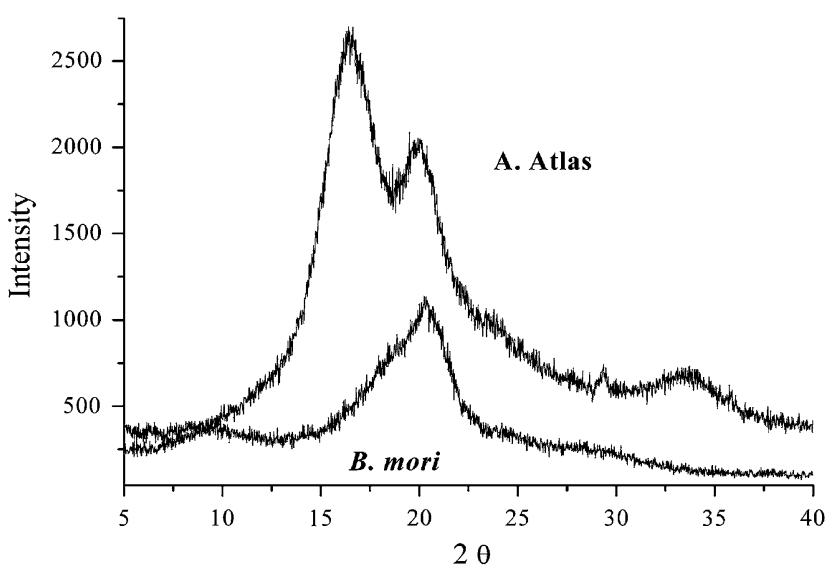

Figure 3. X-ray diffractogram of $A$. atlas fibers compared to $B$. mori silk fibers.

bon-like and have a solid cross-section. The morphology of the $A$. atlas fibers is similar to that of the wild silk but different than B. mori silk that have a triangular cross-section.

\section{Tensile Properties}

The fineness, tensile properties and moisture regain of the silk fibers from the three layers of $A$. atlas cocoons are compared with that of B. mori and two common wild silks in Table 2. As seen from the table, fibers from the outer layer of $A$. atlas cocoons are coarser than the fibers from the intermediate and inner layer. However, the $A$. atlas silk fibers from all the three layers are coarser than B. mori silk fibers but much finer than A. mylitta and $P$. ricini silk fibers. Although the inner layer of $A$. atlas cocoons contains the finest fibers, the breaking tenacity of the fibers in the inner layers $(3.6 \mathrm{~g} / \mathrm{den})$ was lower than the fibers in the outer and fiber layer. The breaking tenacity of the fibers from the outer and intermediate

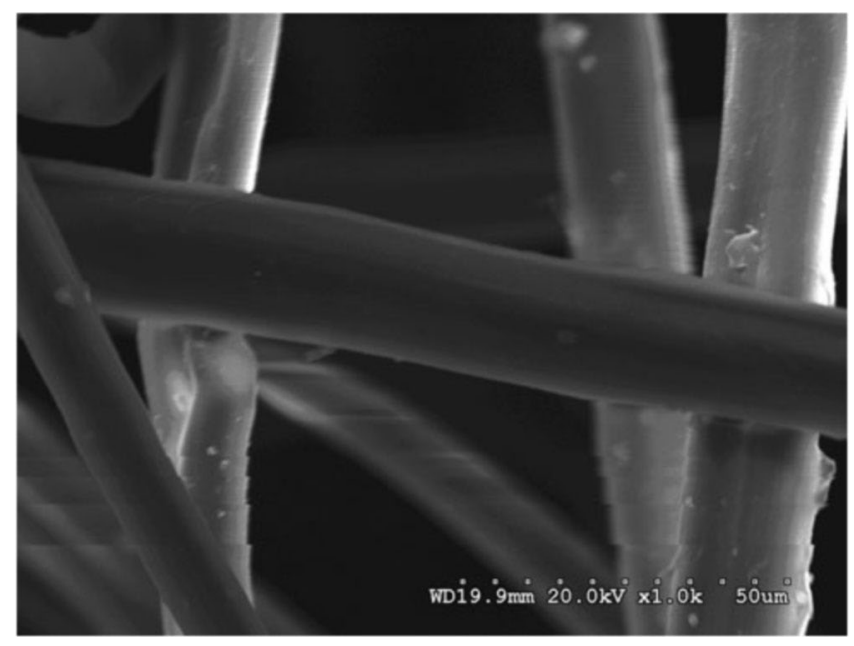

Figure 4. SEM image of $A$. atlas fibers showing the smooth surface of the fibers after degumming.
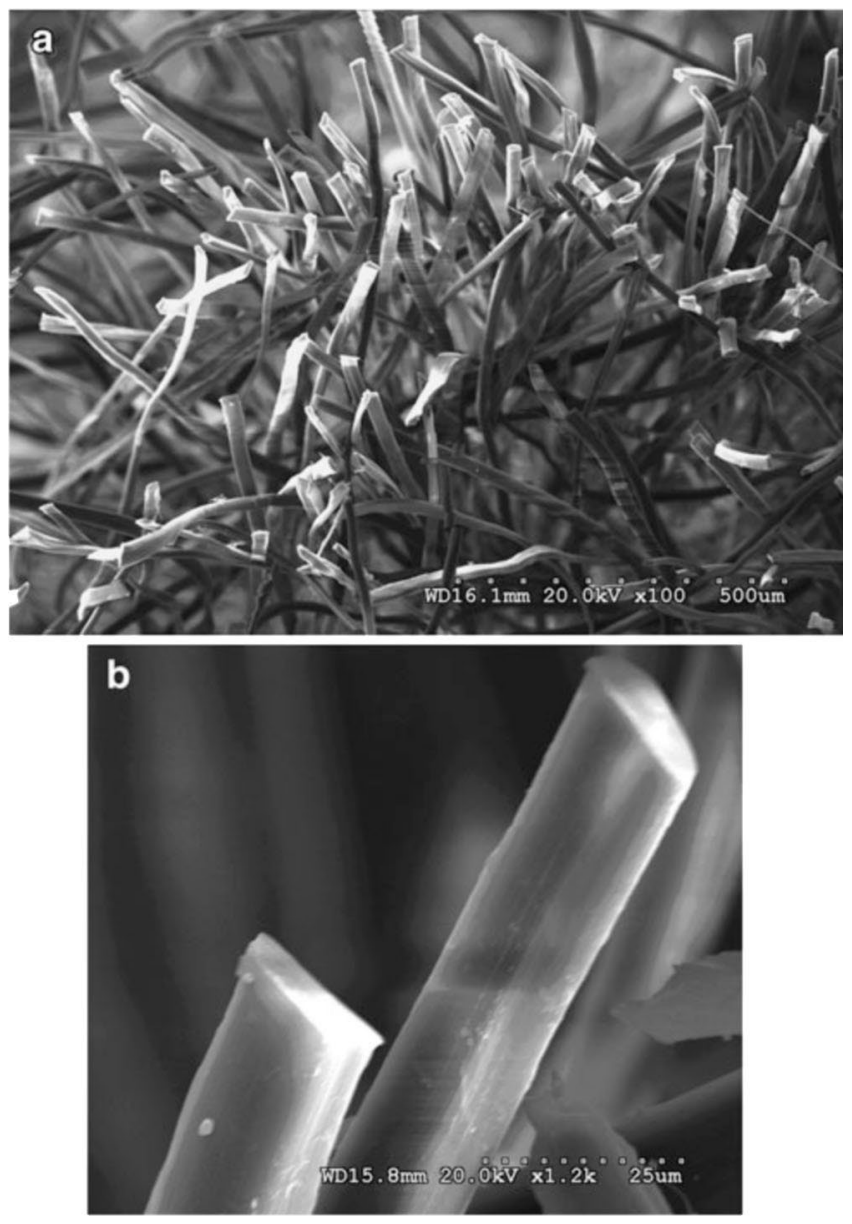

Figure 5. SEM images $\mathrm{a}, \mathrm{b}$ of $A$. atlas fibers showing the rectangular to circular cross-section and smooth surface.

layer were similar to that of B. mori silk and better than that of $P$. ricini silk. The average breaking elongation (15-17.3\%) of the A. atlas fibers was similar to that of $B$. mori silk fibers but lower than that of the wild silks whereas the Young's modulus of the A. atlas silk fibers was lower than that of $B$. mori but higher than that of $P$. ricni silk fibers as seen from the stress-strain curve in Figure 6 . The variations in the tensile properties of the silk fibers from different insects should be due to the differences in the composition, \% crystallinity and arrangement of the crystals in the fibers.

Tensile properties of $A$. atlas silk submerged in liquid media was previously reported [20]. It was reported that immersion in water greatly reduced the elastic modulus but immersion in ethanol increased the modulus. Although $A$. atlas fibers have different amino acid composition compared to that of $B$. mori silk fibers, they have similar mechanical properties. However, the A. atlas fibers have considerably different mechanical properties than spider silk although spider silk and A. atlas have similar amino acid composition [21]. It has also been suggested that the tensile failure mechanism of $A$. 
Table 2. Tensile properties of the silk fibers from the three layers of the A. atlas cocoons compared to a range of tensile properties for B. mori, A. mylitta and P. ricini silks

\begin{tabular}{|c|c|c|c|c|c|c|}
\hline \multirow[t]{2}{*}{ Fiber } & \multicolumn{3}{|l|}{ A. atlas } & \multirow[b]{2}{*}{ B. mori } & \multirow[b]{2}{*}{ A. mylitta } & \multirow[b]{2}{*}{ P. ricini } \\
\hline & Outer & Intermediate & Inner & & & \\
\hline Fineness, denier & 2.7 & 2.0 & 1.7 & $0.4-1.1$ & $4.7-10.7$ & $2.3-3.6$ \\
\hline Strength, g/den & $4.1 \pm 1.5$ & $4.3 \pm 0.8$ & $3.6 \pm 0.6$ & $4.3-5.2$ & $3.9-4.5$ & $1.9-3.5$ \\
\hline Elongation, \% & $17.3 \pm 8.6$ & $18.7 \pm 9.3$ & $15.0 \pm 6.4$ & $10.0-23.4$ & $26-39$ & $24-27$ \\
\hline Modulus, g/den & $53 \pm 18$ & $48 \pm 18$ & $60 \pm 12$ & 84-121 & $66-70$ & $29-31$ \\
\hline Moisture regain, \% & 11.6 & 12.0 & 12.4 & $8.5-9.9$ & 10.5 & 10.0 \\
\hline
\end{tabular}

Data for B. mori and the wild silks are from [11-13]

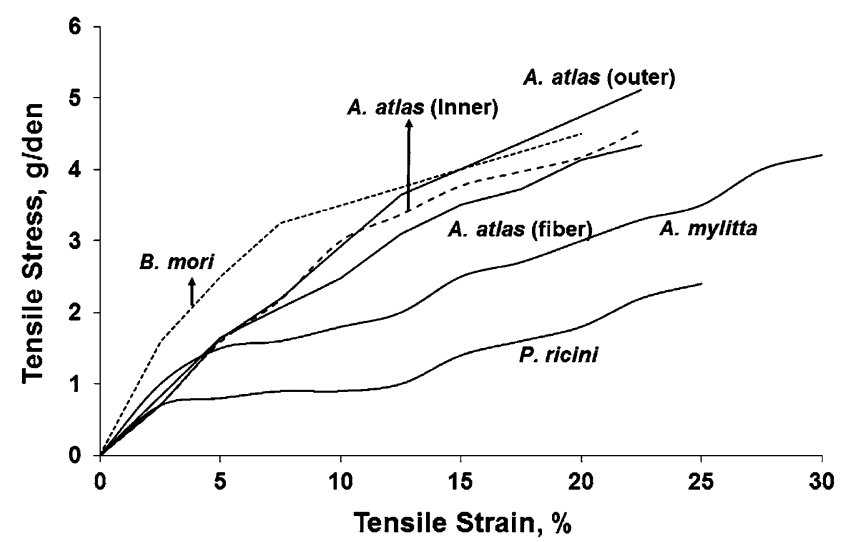

Figure 6. Stress-strain curves of fibers from the three layers in $A$. atlas cocoons compared to B. mori and the common wild silks A. mylitta and $P$. ricini.

atlas fibers involves the pulling of micofibrils from the matrix, similar to that observed in composites [26]. The moisture regain of the $A$. atlas silk fibers was higher than that of the other silk fibers in Table 2. Overall, the tensile properties of the $A$. atlas fibers are similar to that of $B$. mori silk than the common wild silks.

\section{Cell Culture}

Attacus atlas fibers showed considerably higher cell densities from the MTS assay compared to the B. mori silk fibers. Cells on the $A$. atlas fibers reached confluence after 5 days of culture compared to 7 days for the B. mori silks. As seen from Figure 7, the cell density on the $A$. atlas fibers was higher on all days of culture compared to the B. mori fibers. At their respective days of confluence, the $A$. atlas fibers had nearly $80 \%$ higher cell densities per unit area of the fibers suggesting that the $A$. atlas fibers were more conducive to cell attachment and growth. Confocal image in Figure 8 showed that the cells had extensive spreading of the cytoskeleton (red) and the presence of numbers of cells (cell nuclei in blue). The left panel in Figure 8 shows that the cytoskeleton has covered the fibers and the cells appear to grow

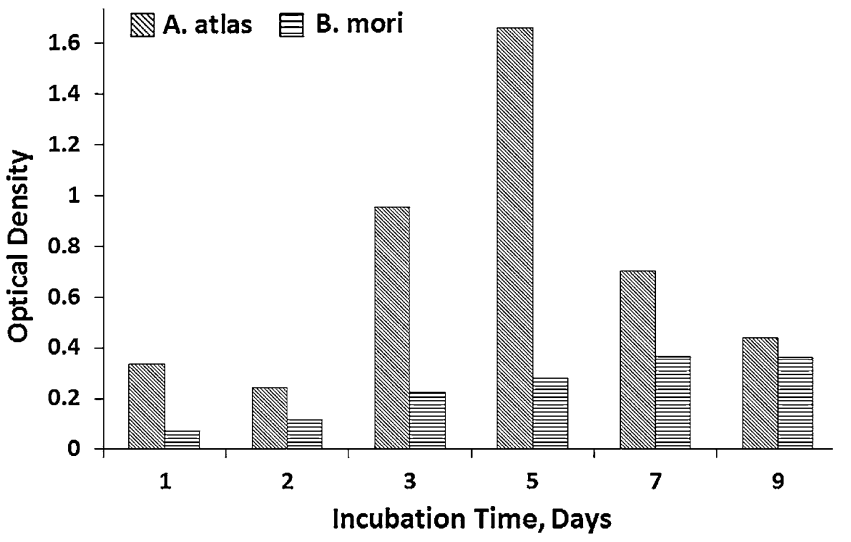

Figure 7. Optical densities per unit weight of the A. atlas and B. mori fibers based on the MTS assay after culture at $37^{\circ} \mathrm{C}$ and $5 \% \mathrm{CO}_{2}$.

along the length of the fibers. Overall, the cell culture studies demonstrate that $A$. atlas fibers are biocompatible and would be suitable for tissue culture and other medical applications.

\section{Conclusions}

Attacus atlas produces considerably heavier cocoons with three distinct layers. The intermediate layer formed the bulk ( $43 \%$ ) of the cocoons whereas the outer and inner layers accounted for 23 and $34 \%$, of the weight of the cocoon, respectively. Degumming was necessary to obtain fibers from the cocoons and the extent of degumming loss decreased from the outer to the inner layers. The amino acid composition in the $A$. atlas fibers was found to be considerably different than the amino acid composition in B. mori silk fibers. Fibers in the outer layer of the cocoons were relatively coarser than the fibers in the intermediate and inner layer. Breaking tenacity (3.6-4.3 g/den) of the fibers from the three layers of $A$. atlas cocoons was similar to that of B. mori silk but much higher than that of the common wild silks. Elongation of the $A$. atlas fibers was similar to that of $B$. mori 

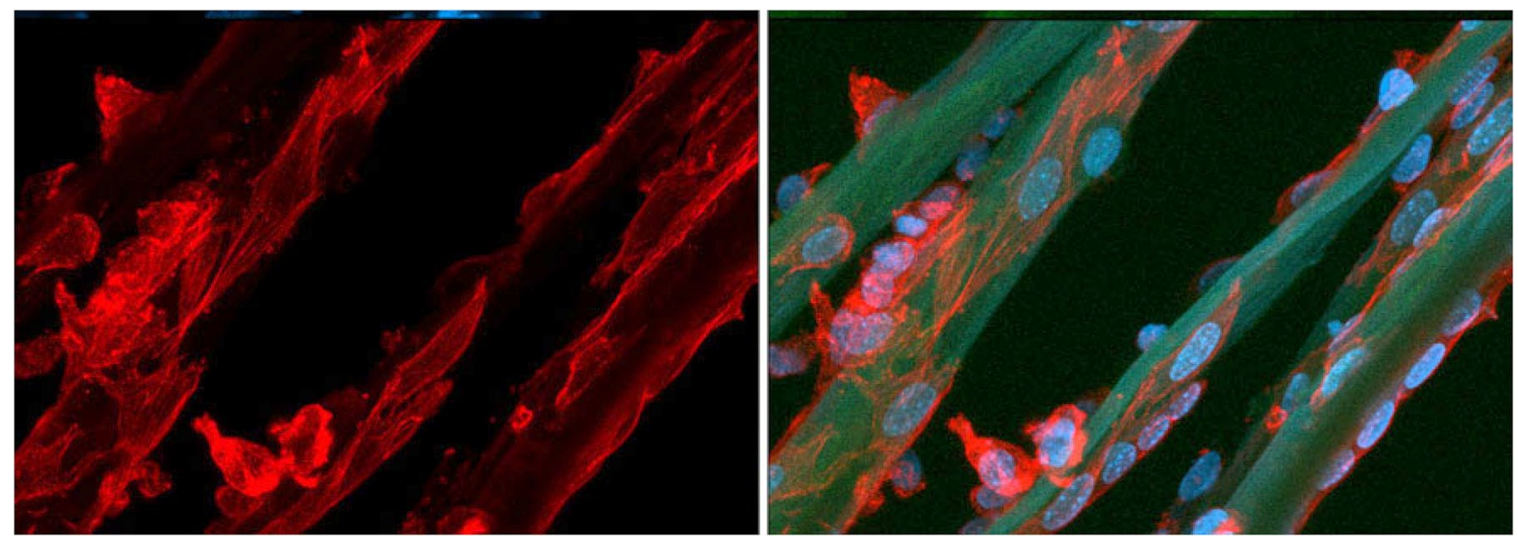

Figure 8. Confocal laser scanning microscope image of the fibroblast cells on A. atlas fibers. The extensive spreading of F-actin is seen in red on the left image and the cell nuclei are seen in blue on the right image.

silk fibers but much lower than that of the wild silks. The $A$. atlas fibers showed considerably higher attachment and proliferation of cells than B. mori silk and extensive growth of F-actin demonstrating that the fibers were biocompatible. Overall, the ability of the A. atlas caterpillars to produce larger cocoons and silk fibers with properties similar to that of $B$. mori silk offers an opportunity to rear the A. atlas as alternative to the common silks for commercial silk production.

Acknowledgments - The authors wish to thank the Agricultural Research Division at the University of NebraskaLincoln, USDA Hatch Act and Multi-state Project S1026 for their financial support to complete this research. The authors also thank Nathan Brockam with Reiman Gardens for his help in collecting the A. atlas cocoons.

\section{References}

1. Zhang X, Reagan MR, Kaplan D, Adv D (2009) Drug Deliv Rev 61:988

2. Mandal BB, Kundu SC (2010) Acta Biomater 6:360

3. Kasoju N, Bhonde RR, Bora U (2009) Mater Lett 63(28):2466

4. Kearns V, MacIntosh AC, Crawford A, Hatton PV (2008) Topics Tissue Eng 4:1

5. Reddy N, Yang Y (2011) Trends Biotechnol 29(10):490

6. Acharya C, Ghosh SK, Kundu SC (2008) J Mater Sci Mater Med 19:2827

7. Acharya C, Ghosh SK, Kundu SC (2009) Acta Biomater $5(1): 429$

8. Seidel A, Liivak O, Calve S, Adaska J, Ji G, Yang Z, Grubb D, Zax DB, Jelinski LW (2000) Macromolecules 33:775
9. Kundu J, Dewan M, Ghoshal S, Kundu SC (2008) J Mater Sci Mater Med 19:2679

10. Zhang Y (2000) Biotechnol Adv 20:91-100

11. Tuskes PM, Tuttle JP, Collins MM (1996) The natural history of the saturniidae of the United States and Canada. Cornell University Press, New York

12. Sen K, Babu MK (2004) J Appl Polym Sci 92:1080

13. Sen K, Babu MK (2004) J Appl Polym Sci 92:1098

14. Robson RM (1992) Silk: composition, structure and properties. In: Lewin M, Pearce EM (eds) Handbook of fiber chemistry. Marcel Dekker Inc, New York, pp 415-456

15. Reddy N, Yang Y (2010) Int J Biol Macromol 46(4):419

16. Reddy N, Yang Y (2010) J Mater Sci 45:4414

17. Reddy N, Yang Y (2010) J Mater Sci 45(24):6617

18. Nurmalitasari G, Kuroda F (2002) 4th international conference on wild silkmoths, April 23-26, Yogyakarta, Indonesia

19. Razafimanantosoa $T$, Ravoahangimalala OR, Craig CL (2006) Madagascar Conserv Dev 1(1):34

20. Kakati LN, Chutia BC (2009) Trop Ecol 50(1):137

21. Perez-Rigueiro J, Elices M, Lloraca J, Viney C (2002) J Appl Polym Sci 82:53

22. Mondal M, Trivedy K, Kumar Caspian SN (2007) J Env Sci 5(2):63

23. Lee Y (1999) Silk reeling and testing manual. FAO, Rome

24. Dash R, Mandal M, Ghosh SK, Kundu SC (2008) Acta Biomater 311:111

25. Padamwar MN, Pawar AP (2004) J Sci Ind Res 63:323

26. Poza P, Perez-Rigueiro J, Elices M, Llorca J (2002) Eng Fract Mech 69:1035 


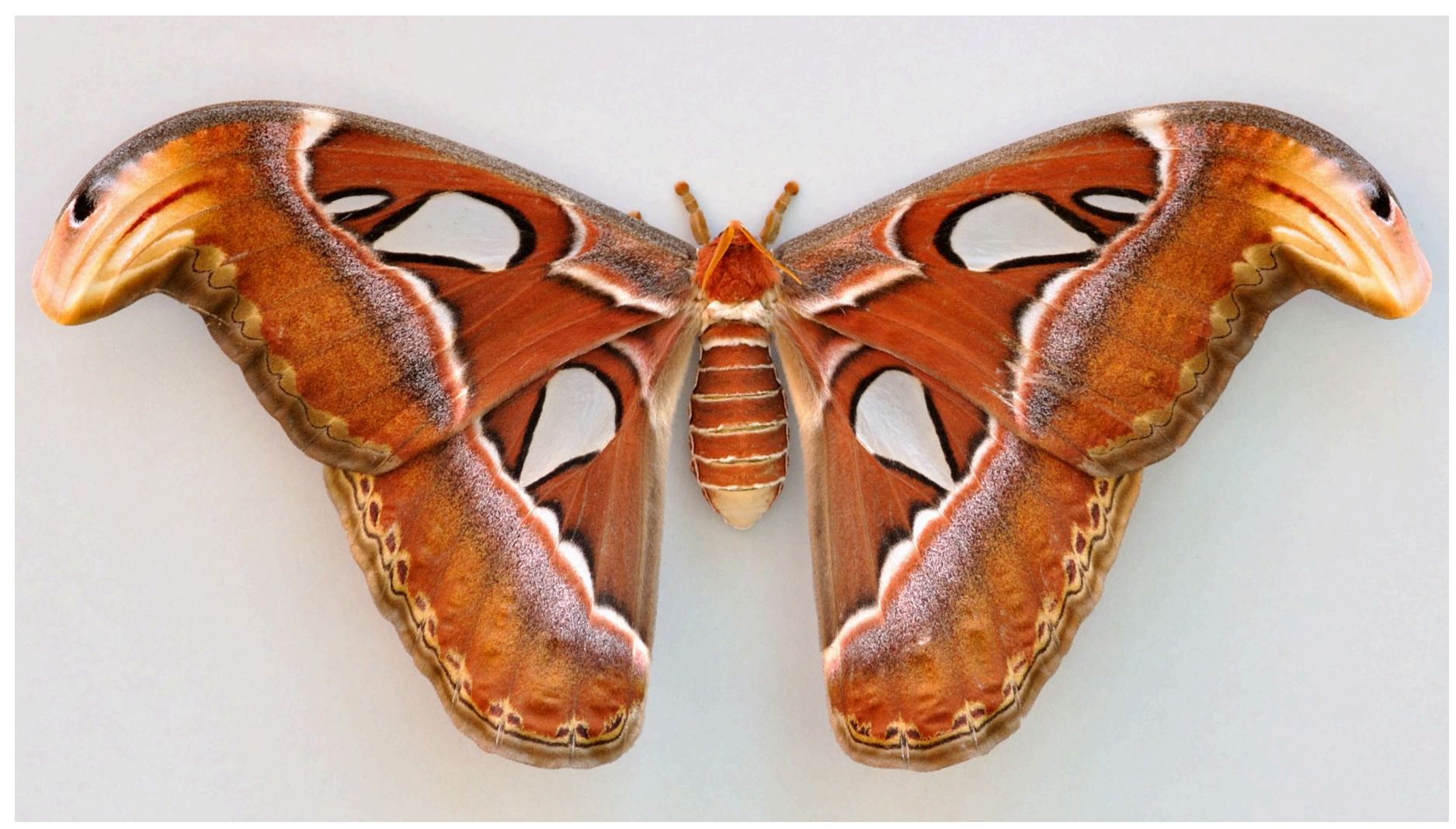

Female Atlas Moth (Attacus atlas) in the Wilhelma, Stuttgart, Germany. This is a live animal sitting high on the back wall of the insectarium laying eggs. The wingspan is roughly $25 \mathrm{~cm}$.

http://en.wikipedia.org/wiki/File:Attacus_atlas_qtl1.jpg

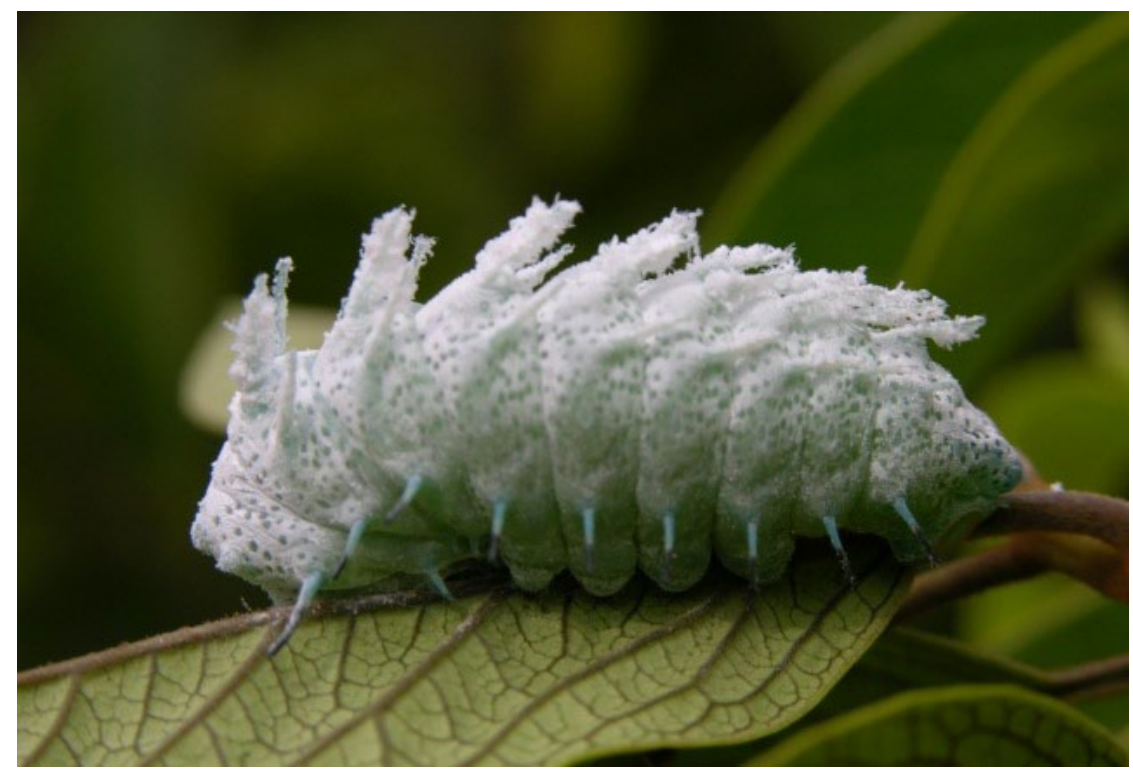

Attacus atlas caterpillar. School of Ecology and Conservation, University of Agricultural Sciences Bangalore; http://commons.wikimedia.org/wiki/File:Attacus_atlas_cat.jpg 